\title{
EVALUATION OF COMPLICATIONS OF FUNCTIONAL ENDOSCOPIC SINUS SURGERY
}

\author{
Liliya Ilieva ${ }^{1}$, Nikolay Sapundzhiev $^{2}$, Georgi Georgiev ${ }^{3}$, Darina Ivanova $^{4}$ \\ ${ }^{1}$ Student, Faculty of Medicine, Medical University of Varna \\ ${ }^{2}$ Department of Neurosurgery and ENT, Faculty of Medicine, Medical University of Varna \\ ${ }^{3}$ Clinic of Otorhinolaryngology, St. Marina University Hospital, Varna \\ ${ }^{4}$ Department of Diagnostic Imaging and Radiotherapy, Faculty of Medicine, \\ Medical University of Varna
}

\begin{abstract}
AIM: The objective of this study is to evaluate the complications after functional endoscopic sinus surgery (FESS) in the Department of Otorhinolaryngology at the St. Marina University Hospital, Varna.

MATERIALS AND METHODS: The records of all patients subjected to FESS were evaluated retrospectively. The complications were classified as major (severe hemorrhage, leakage of cerebral spinal fliud, visual disturbances) and minor (mild hemorrhage, periorbital hematoma and cellulitis, subcutaneous emphysema, epiphora).

RESULTS: The records of all patients subjected to FESS in the time period March 2006 to January 2016 were retrieved from the digital patient record system of a single institution, St Marina University Hospital, in Varna, Bulgaria and evaluated retrospectively. A total of 65 patients aged 11-77 (2 children, mean age $13 \pm 2.8$ years and 63 adults, mean age $46.5 \pm 13.9$ years, 41 men and 24 women), who were subjected to FESS, were evaluated. Ten cases were classified as minor complications $(15.3 \%)$. They included subcutaneous emphysema $(n=1,1.5 \%)$ and minimal postoperative bleeding $(n=9,13.8 \%)$. Four cases were classified as major complications (6.1\%). They included severe hemorrhage, requiring transfusion, nasal packing or rehospitalization $(n=3,4.6 \%)$, and visual disturbances $(n=1,1.5 \%)$.

CONCLUSION: FESS may be considered a relatively safe method with low rate of the complications, but as with any surgical intervention, it carries risks. According to other published reports the average rate of minor and major FESS complications is about $9 \%$ and about $0.9 \%$, respectively.
\end{abstract}

Keywords: FESS, functional endoscopic sinus surgery, complications

Address for correspondence:

Nikolay Sapundzhiev

Department of Neurosurgery and ENT,

Faculty of Medicine,

Medical University of Varna

55 Marin Drinov St

9002 Varna

e-mail:n.sapundzhiev@mu-varna.bg

Received: April 1, 2018

Accepted: June 29, 2018

\section{INTRODUCTION}

Functional endoscopic sinus surgery (FESS) is a minimally invasive, sophisticated surgical technique, using an endoscope or microscope to restore the drainage and ventilation of the nasal cavity and paranasal sinuses. It has been used for over 20 years to treat diseases of the nose and the paranasal cavities. The most common indications for FESS are: chronic sinusitis refractory to medications and polyps in the nasal cavity $(1,2)$. 
As with any invasive treatment modality an extensive list of complications has been reported in the literature so far. The major complications associated with FESS include severe hemorrhage, leakage of cerebral spinal fliud, and visual disturbances. Examples of minor complications are mild hemorrhage, periorbital hematoma and cellulitis, subcutaneous emphysema, and epiphora. Most orbital complications are classified as major because of the potential loss of vision, but orbital emphysema is considered a minor complication $(2,3)$.

\section{AIM}

The objective of the present study is to evaluate the complications after FESS in the Department of Otorhinolaryngology at the St. Marina University Hospital, Varna.

\section{MATERIALS AND METHODS}

The records of all patients subjected to FESS in the time period March 2006 to January 2016 were retrieved from the digital patient record system of a single institution, St Marina University Hospital in Varna, Bulgaria and evaluated retrospectively (4). With regard to patient characteristics, information on patients' birth date and examination date was included in the file indexing system, and the sex of each patient was contained in their personal identification number. The complications were classified as major

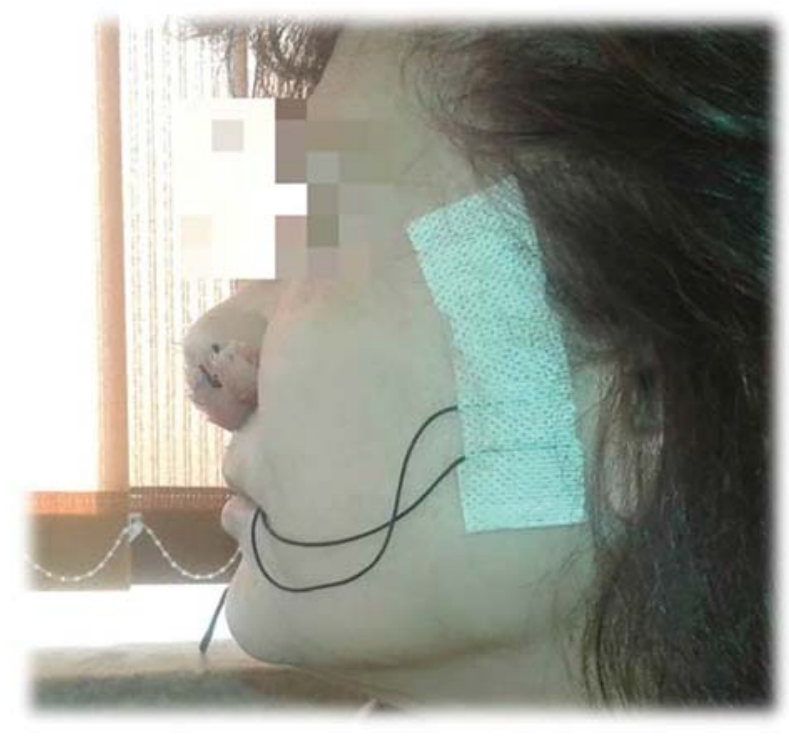

(severe hemorrhage, leakage of cerebral spinal fliud, and visual disturbances) and minor (mild hemorrhage, periorbital hematoma and cellulitis, subcutaneous emphysema, and epiphora).

\section{RESULTS}

A total of 65 patients aged 11-77 (2 children, mean age $13 \pm 2.8$ years and 63 adults, mean age $46.5 \pm 13.9$ years, 41 men and 24 women), who were

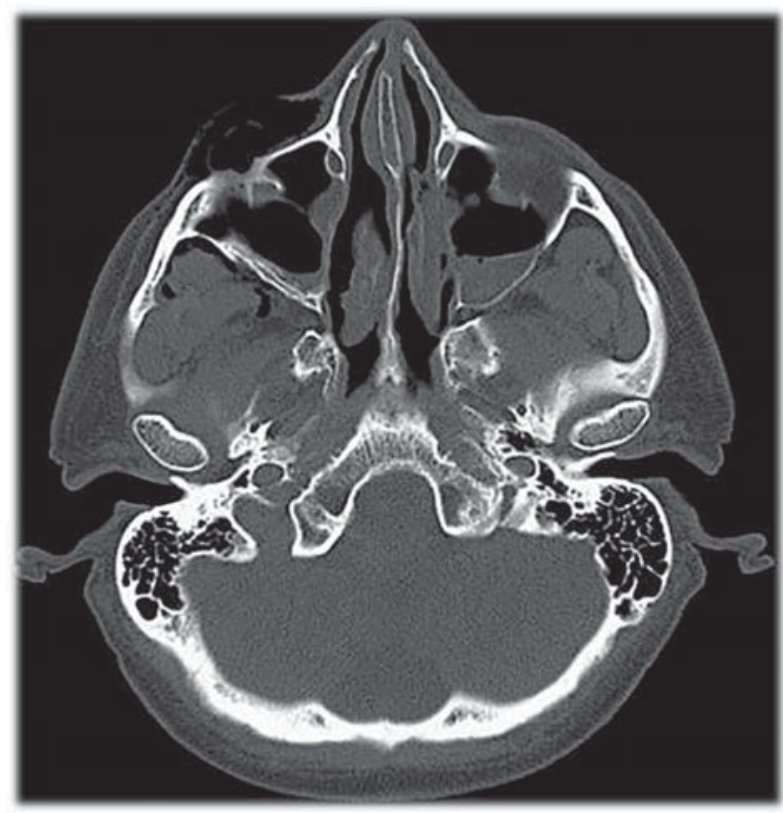

Fig. 1. Computed tomography of a patient with subcutaneous periorbital emphysema

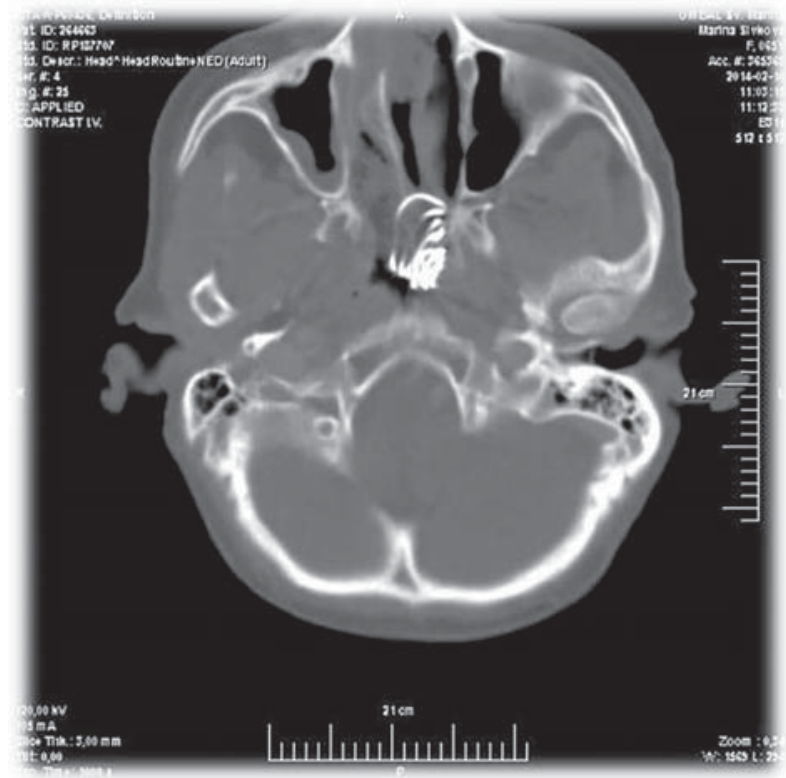

Fig. 2. A. A patient with anterior and posterior nasal packing. B. Computed tomography of a patient with nasal packing 
Liliya Ilieva, Nikolay Sapundzhiev, Georgi Georgiev et al.

subjected to FESS, were evaluated. The indications for surgery included nasal polyposis $(56.9 \%, 37 / 65)$, chronic sinusitis $(21.5 \%, 14 / 65)$, polyposis combined with sinusitis $(9.2 \%, 6 / 65)$, benign tumors $(10.7 \%$, $7 / 65)$ and malignant tumors $(1.5 \%, 1 / 65)$. The types of FESS interventions performed included polypectomy (10.9\%), endoscopic incision of multiple sinuses $(29.6 \%)$, combined interventions (48.4\%), and excision/extraction of other lesions (10.9\%). Ten cases were classified as minor complications (15.3\%). They included subcutaneous emphysema $(\mathrm{n}=1,1.5 \%)$, (Fig. 1) and minimal postoperative bleeding $(n=9,13.8 \%)$, (Fig. 2A, 2B). Four cases were classified as major complications (6.1\%). They included severe hemorrhage, requiring transfusion, nasal packing or rehospitalization $(n=3,4.6 \%)$, and visual disturbances $(\mathrm{n}=1,1.5 \%)$.

\section{DISCUSSION}

FESS is considered a safe and standard method for treatment of the nasal and paranasal cavities, but the statistics show that complications can occur. According to literature reviews the average rate of minor complications is between $2.6 \%(9 / 260)$ and $10.5 \%$ $(28 / 266)(5,6)$. The complication rate of minimal postoperative bleeding is described to be $2 \%(6 / 300)$ and the scientific data about cases with subcutaneous emphysema is $0.1 \%$ (4/3402). (7), (8). The overall major complications rate according to published reports is between $0.36 \%(288 / 78944)$ and $1 \%(630 / 62$ $823)$, including severe hemorrhage requiring transfusion or nasal packing $(0.14 \%, 75 / 50734$ to $0.76 \%$, $478 / 62823)$ and orbital injury - $0.07 \%(45 / 62823)$ to $0.7 \%(25 / 3,402)(1,7,9,10)$. Sayaka Suzuki et al. reported a rate of $0.02 \%(12 / 50734)$ for the cases with toxic shock syndrome (1). The literature-based results about leakage of cerebral spinal fluid are between $0.09 \%(50 / 50734)$ and $0.17 \%(108 / 62823)(1,10)$.

The results of our group of patients show higher complications rate. This may be attributed to the surgical experience. Another potential factor may be the equipment available. Still, we believe that it is the design and the focus of our study that led to this bias. We deliberately looked for and analyzed the records for any appearance of the word "bleeding", even if it was not considered/coded as a complication at the time of discharge or it did not change the clinical and ambulatory follow-up of the patient.
In Bulgaria the patients are often being informed that FESS is a "bloodless" intervention, but obviously that is not true. The operation carries the risk of postoperative bleeding that depends on the type and extent of the intervention (polypectomy, tumorectomy, FESS for chronic sinusitis). The patients should be informed about the eventual postoperative complications.

According to the literature review, post-operative hemorrhages are classified depending on the type of bleeding and the moment when they start. Depending on the bleeding source the post-operative hemorrhage is arterial, capillary, and venous. The second classification includes 3 main groups: primary, reactive, and secondary bleeding. Primary bleeding occurs in the intra-operative period, reactive - in the first 24 hours after the operation and the secondary - 7-14 days postoperatively. In the group of patients, who underwent FESS in our institution, there were 2 cases with primary and 5 cases with reactive bleeding. None of them led to significant change in the postoperative follow-up or prolongation of the hospital stay.

The most common purpose of nasal packing is for bleeding control after surgery of the nasal cavity. Nasal packing is done routinely in the majority of the patients, who are subjected to FESS. Basha S. I. et al. made a retrospective analysis of 110 patients who underwent nasal operations and evaluated the complication rate when nasal packs were not routinely inserted. Nine cases needed nasal packing at the end of the surgery and 4 cases - in the postoperative period (11). According to our study, all of the patients had nasal packing after surgery. The results show that one patient needed hemotransfusion and one patient needed repacking - both cases with secondary (late) bleeding after discharge.

\section{CONCLUSION}

FESS may be considered a relatively safe method with a low rate of complications, but as with any surgical intervention, it carries risks. According to other published reports the average rate of minor complications is about $2.6 \%$ to $10.5 \%$ and the rate of major is about $0.36 \%$ to $1 \%(5,6,9,10)$. FESS is one of the most commonly performed operations in otorhinolaryngology and is generally a safe procedure, but major complications may occur. 


\section{REFERENCES}

1. Suzuki S, Yasunaga H, Matsui H, Fushimi K, Kondo K, Yamasoba T. Complication rates after functional endoscopic sinus surgery: analysis of 50,734 Japanese patients. Laryngoscope. 2015;125(8):178591. doi: 10.1002/lary.25334.

2. Functional Endoscopic Sinus Surgery: Overview, Preparation, Technique. 2016 Jun 28 [cited 2016 Oct 30]; Available from: http://emedicine.medscape.com/article/863420-overview

3. Functional Endoscopic Sinus Surgery for Sinusitis [Internet]. Medscape. [cited 2016 Oct 31]. Available from: http://www.medscape.com/ viewarticle/715536

4. Sapundzhiev N. Digital oncology patient record - heterogeneous file based approach. Journal of IMAB - Annual Proceeding (Scientific Papers). 2010; 16(3):40-3. doi:10.5272/jimab.1632010_40-43

5. Bajaj Y, Gadepalli C, Reddy TN. Functional Endoscopic Sinus Surgery: Review Of 266 Patients. Internet J Otorhinolaryngol [Internet]. 2006 Dec 31 [cited 2016 Oct 31];6(1). Available from: http://ispub.com/IJORL/6/1/8172

6. Närkiö-Mäkelä M, Qvarnberg Y. Endoscopic sinus surgery or Caldwell-Luc operation in the treatment of chronic and recurrent maxillary sinusitis. Acta Oto-Laryngol Suppl. 1997;529:177-80.

7. Stankiewicz JA, Lal D, Connor M, Welch K. Complications in endoscopic sinus surgery for chronic rhinosinusitis: a 25-year experience. Laryngoscope. 2011;121(12):2684-701. doi: 10.1002/lary.21446.

8. Jonnalagadda $\mathrm{S}, \mathrm{Yu}$ VM, Catalano P. Endo-nasal surgeries without nasal packing or splints - A retrospective review of postoperative bleeding complications in 300 patients. The Laryngoscope. 2011;121(S5):S362-S362. doi: 10.1002/lary.22318.

9. Krings JG, Kallogjeri D, Wineland A, Nepple KG, Piccirillo JF, Getz AE. Complications of primary and revision functional endoscopic sinus surgery for chronic rhinosinusitis. Laryngoscope. 2014;124(4):838-45. doi: 10.1002/lary.24401.

10. Ramakrishnan VR, Kingdom TT, Nayak JV, Hwang PH, Orlandi RR. Nationwide incidence of major complications in endoscopic sinus surgery. Int Forum Allergy Rhinol. 2012;2(1):34-9. doi: 10.1002/alr.20101.

11. Basha SI, Gupta D, Kaluskar SK. Routine nasal packing follwoing nasal surgery-Is it necessary? Indian J Otolaryngol Head Neck Surg. 2005;57(1):6971. doi: 10.1007/BF02907637. 